\title{
Summary of the Public Health Agency of Canada's Action Plan on Lyme Disease
}

\author{
Harymann $\mathbf{M}^{1}$, Ogden $\mathbf{N}^{1}$, Lindsay $\mathbf{R}^{1}$, Lawless $\mathbf{V}^{1}$, Deilgat $\mathbf{M}^{1}$ and Sternthal $\mathbf{S}^{1}$ \\ ${ }^{1}$ Public Health Agency of Canada, Centre for Food-borne, Environmental and Zoonotic Infectious Diseases \\ * Corresponding author: marian.harymann@phac-aspc.gc.ca
}

\section{Abstract}

Background: Lyme disease is an emerging infectious disease in Canada that requires a comprehensive approach to prevention and control. It is a serious illness caused by a bacterium transmitted by certain types of ticks. The risk of Lyme disease currently exists in southern parts of British Columbia and Manitoba, southern and eastern Ontario, southern Quebec and New Brunswick, and in some locations in Nova Scotia.

Objective: To highlight the Public Health Agency of Canada's Action Plan on Lyme Disease, which aims to mitigate the risks to Canadians posed by Lyme disease through concrete activities being undertaken jointly with the provinces, territories, and various stakeholders.

Approach: A multidisciplinary approach was used to assess the evidence on Lyme disease in Canada, analyze stakeholder concerns and evaluate what was currently available to inform public health professionals and the public. This assessment informed the development of an action plan intended to address the prevention, diagnosis and treatment of Lyme disease.

Results: The Action Plan on Lyme Disease sets out concrete action to be undertaken over three years, beginning in March 2014. It is built upon three pillars: 1.) Engagement, education and awareness, 2.) Surveillance, prevention and control, and 3.) Research and diagnosis.

Conclusion: Effective prevention and control of Lyme disease in Canada requires a coordinated multi-partner and stakeholder engagement approach.

\section{Introduction}

Lyme disease is an emerging infectious disease in Canada that requires a comprehensive approach to prevention and control. It is a serious illness caused by a bacterium transmitted by certain types of ticks. The risk of Lyme disease currently exists in southern parts of British Columbia and Manitoba, southern and eastern Ontario, southern Quebec and New Brunswick, and in some locations in Nova Scotia. As migratory birds and other animal hosts carry ticks to new areas, the risk of Lyme disease is spreading. To promote prevention, early diagnosis, and treatment, greater public awareness of the disease is needed along with collaborative engagement of public health and health care professionals, academics, scientists, and patient advocacy groups.

The objective of this Action Plan is to mitigate the risks to Canadians posed by Lyme disease through concrete activities being undertaken jointly with the provinces, territories, and various stakeholders.

\section{Approach}

A multidisciplinary approach was used to assess the surveillance, risk assessment, and research evidence on Lyme disease in Canada and internationally; to analyze diverse stakeholder concerns regarding prevention, diagnosis and treatment of the disease; and to evaluate the usefulness of information available to public health professionals and the public. This information was then used to inform the development of an action plan intended to address the prevention of Lyme disease, so that any Lyme disease cases that do occur can be diagnosed and 
treated early in the course of disease. The plan was also aligned with priority areas of the Public Health Agency of Canada and key federal objectives (Figure 1).

\section{The Action Plan}

The Action Plan is built upon three pillars: 1.) Engagement, education and awareness, 2.) Surveillance, prevention and control, and 3.) Research and diagnosis.

\section{Engagement, education and awareness}

A key element in preventing Lyme disease and promoting early diagnosis and treatment is raising public awareness and healthcare provider knowledge. To achieve this goal, activities will focus on:

- An advertising campaign targeting health care professionals, as well as Canadians practising outdoor activities;

- Stakeholder outreach, e.g. the dissemination of a comprehensive toolkit of educational materials for a range of end users, including public health professionals;

- Media engagement, including proactive interviews with experts and a partnership with News Canada;

- Social media activities, including Facebook, Twitter, and blogs.

The Public Health Agency of Canada (the Agency) is actively engaging provincial and territorial health authorities and other stakeholders to coordinate a national communications response to Lyme disease to better protect Canadians.

\section{Surveillance, prevention and control}

The collection, analysis, and interpretation of epidemiological data are essential features of public health practice. The Agency has developed an approach to national surveillance for both ticks and human cases of Lyme disease. The surveillance aims to measure national and regional changes in incidence and to identify populations at risk. To address the need for continued and enhanced surveillance, prevention, and control for Lyme disease, the Agency is:

- Working with partners to explore innovative ways to conduct surveillance;

- Developing strategies to encourage preventive behaviour;

- Conducting systematic reviews and other research on the epidemiology, prevention, and control of Lyme disease in Canada to inform the development of public health guidelines;

- Undertaking field studies to inform risk models that identify emerging risk areas.

The Agency also plans to consult with stakeholders to help improve prevention efforts, and public health guidelines on Lyme disease to reflect the latest scientific evidence and best practices.

\section{Research and diagnosis}

There is a need for continued research on Lyme disease and other emerging tick-borne diseases to better understand the causes and complications of the disease, and how to effectively diagnose and treat it. Through examination of evidence-based research, the Agency will explore:

- New methods of controlling the ticks that carry and spread Lyme disease;

- Improved diagnostic methods as they become available;

- The strains and species of tick-borne pathogens, their geographic locations, and their possible implications for diagnosis and disease severity.

Clinical practice and laboratory guidelines will be reviewed and updated. 
Figure 1: The three pillars of the Action Plan on Lyme Disease and their alignment with priority areas, key federal objectives, goals, and vision

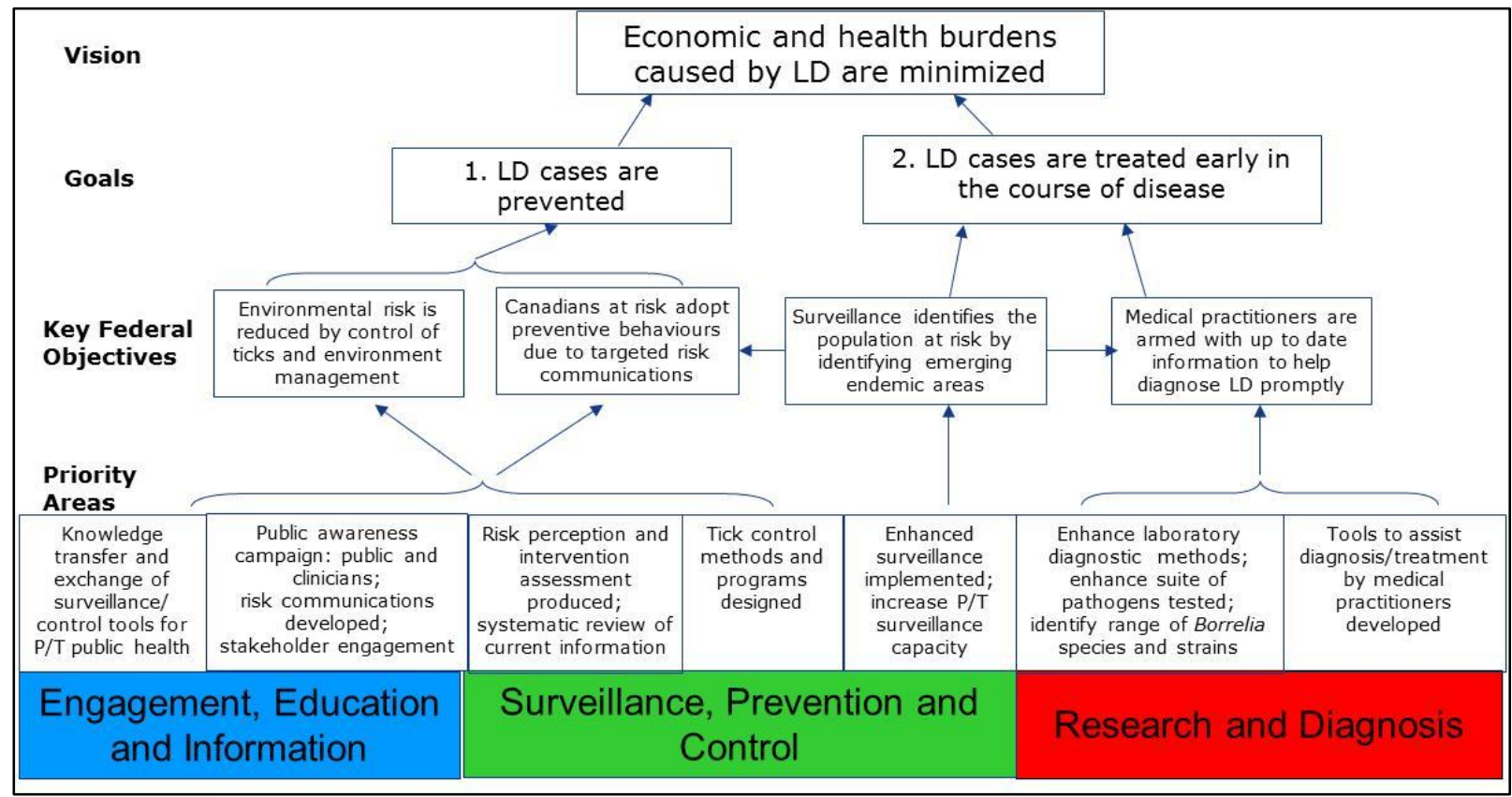

\section{Conclusion}

Effective prevention and control of Lyme disease in Canada requires a coordinated multi-partner and stakeholder engagement approach. This will contribute to minimizing the impact of Lyme disease through:

- Improved understanding and awareness of Lyme disease by the public, health care providers, and other stakeholders;

- Enhanced national surveillance to pinpoint where the disease is emerging and which populations are at risk;

- Research to generate new insights into effective diagnosis and treatment;

- Promotion of early diagnosis and treatment of Lyme disease.

\section{Acknowledgements}

We recognize the input of many stakeholders who contributed to the development of this Action Plan and who will continue to be vital in its implementation. Many thanks to the individuals in the Public Health Agency of Canada who also provided input into drafts of the Action Plan.

\section{Conflict of interest statement}

There are no conflicts of interest to declare.

\section{Funding}

This work was supported by the Public Health Agency of Canada.

\section{References}

Public Health Agency of Canada. Action Plan on Lyme disease.

http://www.phac-aspc.gc.ca/id-mi/lyme-plan-eng.php 